\title{
Short and mid-term reproducibility analysis of cerebral tissue saturation measured by time domain-NIRS
}

F. Lange, I. Tachtsidis

F. Lange, I. Tachtsidis, "Short and mid-term reproducibility analysis of cerebral tissue saturation measured by time domain-NIRS," Proc. SPIE 11074, Diffuse Optical Spectroscopy and Imaging VII, 110740V (11 July 2019); doi: $10.1117 / 12.2526780$ 


\title{
Short and mid-term reproducibility analysis of cerebral tissue saturation measured by time domain-NIRS
}

\author{
F. Lange*a, I. Tachtsidis ${ }^{\mathrm{a}}$ \\ ${ }^{a}$ Department of Medical Physics and Biomedical Engineering, University College London, Malet \\ Place Engineering Building, Gower Street, London WC1E 6BT, UK \\ *f.lange@ucl.ac.uk
}

\begin{abstract}
The short- (i.e. over minutes) and mid-term (i.e. over days/weeks) reproducibility of cerebral tissue saturation measured by time domain-NIRS (TD-NIRS) is investigated in this work. We present a pilot study that assess the reproducibility of the measurement of the tissue saturation $\left(\mathrm{StO}_{2}\right)$ of the prefrontal cortex on a sample of 7 healthy adult volunteers. TDNIRS measurements were acquired at 16 wavelengths, from 780 to $870 \mathrm{~nm}$, in steps of $6 \mathrm{~nm}$, and were fitted with the diffusion model for semi-infinite homogenous media. Then the absolute concentration of oxy- and deoxyhemoglobin $\left(\left[\mathrm{HbO}_{2}\right],[\mathrm{HHb}]\right)$ were calculated using Beer-Lambert's law, in order to calculate the volunteer's brain tissue saturation $\left(\mathrm{StO}_{2}=\left[\mathrm{HbO}_{2}\right] /\left(\left[\mathrm{HbO}_{2}\right]+[\mathrm{HHb}]\right)\right)$. Three measurement sessions were performed on three different days (within a week interval) to evaluate the mid-term reproducibility of the $\mathrm{StO}_{2}$. For each session, three measurements were taken (within 10 minutes, with repositioning of the probes) to evaluate the short-term reproducibility of the $\mathrm{StO}_{2}$. The reproducibility was expressed as the within-volunteer standard deviation $\left(\mathrm{SD}_{\mathrm{w}}\right)$, calculated using a one-way repeated-measure ANOVA. The $\mathrm{SD}_{\mathrm{w}}$ in session 1,2 and 3 were $0.89 \%, 1.10 \%$ and $0.82 \%$ respectively, showing a good short-term reproducibility, and the $\mathrm{SD}_{\mathrm{w}}$ for all 3 sessions was $1.43 \%$, showing a good mid-term reproducibility without significant variations in the $\mathrm{StO}_{2}$ between the 3 sessions. Moreover, the mean $\pm \mathrm{SD}$ global values of $\mathrm{StO}_{2}$ over all the measurements $(\mathrm{n}=63)$ is $62.8+/-4.2 \%$ which is close to the values reported in the literature for adults.
\end{abstract}

Keywords: Times domain NIRS, Brain oximetry, Brain tissue saturation, Photon migration, Light propagation in tissues, Tissue characterization, Blood or tissue constituent monitoring, Medical optics instrumentation.

\section{INTRODUCTION}

Near-infrared spectroscopy (NIRS) is a tool that is able to measure non-invasively, at the bed-side, and continuously, parameters related to cerebral hemodynamics and metabolism ${ }^{1}$. The most widespread NIRS technique to retrieve these parameters is continuous-waves (CW) NIRS. This technique has been around for more than 30 years, with many commercial devices available. However, despite this long history, NIRS still struggles to establish itself as a valuable neuromonitoring tool in the clinic, due to inherent limitations such as depth sensitivity, quantification and reproducibility ${ }^{2}$. Other, NIRS techniques such as frequency-domain (FD) and time-domain (TD), have enabled us to overcome some of these limitations. Particularly, the use of TD-NIRS allows us to calculate the absorption and scattering coefficients of the tissue, enabling us to retrieve absolute concentrations of the chromophores of interest ${ }^{3}$. Of those chromophores, oxy- and deoxyhemoglobin $\left(\mathrm{HbO}_{2}\right.$ and $\left.\mathrm{HHb}\right)$ can be used to estimate the tissue saturation $\left(\mathrm{StO}_{2}=\left[\mathrm{HbO}_{2}\right] /\left(\left[\mathrm{HbO}_{2}\right]+[\mathrm{HHb}]\right)\right)$. This is often measured with commercial brain oximeters at the patient bedside and used as an indicator of absolute brain tissue oxygenation; which can inform the patient's brain health status ${ }^{4,5}$.

In order to become a valuable neuromonitoring tool, NIRS instruments need to produce reliable data over time, and some standards need to be set to compare the results. Recently, efforts to standardize the measurements performed with TDNIRS systems have increased, and well-defined protocols have emerged ${ }^{6}$. These protocols allow us to compare the basic performance of the systems, as well as the accuracy of the extracted absorption and scattering coefficients measured, which will then be used to extract $\mathrm{StO}_{2}$. These protocols constitute the first step of standardization, for the technology to be globally adopted. The next steps, in the context of bedside monitoring of brain oxygenation, are to assess the in-vivo reproducibility of the measurements and define normal physiological values on healthy subjects. A recent study investigated this with TD-NIRS and reported good reproducibility results over a short time period (i.e. over. minutes) ${ }^{7}$. In addition, a study by Hallacoglu and collegeues ${ }^{8}$ with FD-NIRS reported a good long-term (i.e. over months) reproducibility. The aim of our study is to assess on healthy adult volunteers, both the short-term (i.e. over minutes) and the mid-term (i.e. over days/weeks) reproducibility of $\mathrm{StO}_{2}$ with our 16 wavelength TD-NIRS instrument.

Diffuse Optical Spectroscopy and Imaging VII, edited by Hamid Dehghani, Heidrun Wabnitz, Proc. of SPIE-OSA Vol. 11074, 110740V · (c) 2019 SPIE-OSA · CCC code: 1605-7422/19/\$21 · doi: 10.1117/12.2526780 


\section{METHODS}

The TD-NIRS instrument used in this study, named MAESTROS, has been reported in a previous publication ${ }^{11}$. Essentially, MAESTROS is based on a supercontinuum laser coupled with an acousto-optical tunable filter that permits the selection of 16 narrow wavelengths, with a full width at half maximum between 2 and $4 \mathrm{~nm}$, in the range of 650 to $1100 \mathrm{~nm}$. The light is then transmitted to the tissue via a single core optical fibre. The system allows up to two sources points. On the detection side, four optical fibres collect the reflected light to four photon multiplier tubes (PMTs). Then, a router is used to redirect the signal to a single TCSPC card, in order to measure the arrival time of the photons.

Seven healthy volunteers (age 26-31 years, 4 females) were recruited from the lab and informed consent was obtained, in conformity with the UCL Local Research Ethics. Measurements were performed on the left forehead of the volunteers sitting in a chair, with the probed located $3 \mathrm{~cm}$ over the eyebrow line. One source and one detector were used in this study. The emitter and receiver fibers were separated by a $3.0 \mathrm{~cm}$ distance using a custom, hand hold, 3D printed probe holder. Measurements were performed in a dark room to decrease the amount of background light. Time-resolved reflectance measurements were simultaneously performed at 16 wavelengths, from 780 to $870 \mathrm{~nm}$, every $6 \mathrm{~nm}$. The total exposure time per wavelength was optimized to get at least $10^{6}$ photons for each wavelength. The total time to acquire the 16 wavelengths in these conditions never exceeded $1 \mathrm{~min}$. This procedure was repeated 3 times, after removing and repositioning the probe holder (short-term reproducibility); with 1 minute between each measurement. After each session, an instrument response function (IRF) was acquired. The present abstract reports the results of 3 sessions, recorded within a week between the first and the last session, with only 1 session measured per day.

Regarding the data processing, TD-NIRS data at each wavelength were fitted with the solution of the diffusion equation for semi-infinite homogenous media after convolution with the IRF. Then, we used Beer-Lambert's law to express the absorption coefficient as a linear combination of the specific absorption of $\mathrm{HbO}_{2}, \mathrm{HHb}$ and water, with $\mathrm{HbO}_{2}$ and $\mathrm{HHb}$ as free parameters and water content fixed to $80 \%{ }^{7}$. Finally, the $\mathrm{StO}_{2}$ was calculated from the $\mathrm{HbO}_{2}$ and $\mathrm{HHb}$ concentrations. All the data processing was done offline, with MATLAB version R2018a.

\section{RESULTS}

Table 1. Calculated $\mathrm{StO}_{2}$ values for each volunteer and each session. Values are reported as: mean (+/- standard deviation (SD)).

Volunteer $\quad \mathrm{StO}_{2}$ session $1(n=3) \quad \mathrm{StO}_{2}$ session $2(n=3) \quad \mathrm{StO}_{2}$ session $3(n=3) \quad$ Average $\mathrm{StO}_{2}(n=9)$

\begin{tabular}{c|lll|l}
\hline 1 & $58.6 \%(+/-0.6 \%)$ & $59.2 \%(+/-0.6 \%)$ & $63.1 \%(+/-1.0 \%)$ & $60.3 \%(+/-2.2 \%)$ \\
2 & $60.4 \%(+/-1.2 \%)$ & $61.7 \%(+/-0.8 \%)$ & $63.2 \%(+/-0.4 \%)$ & $61.8 \%(+/-1.4 \%)$ \\
3 & $64.0 \%(+/-0.5 \%)$ & $64.0 \%(+/-0.9 \%)$ & $63.3 \%(+/-1.1 \%)$ & $63.8 \%(+/-0.9 \%)$ \\
4 & $56.3 \%(+/-1.0 \%)$ & $56.7 \%(+/-2.2 \%)$ & $56.6 \%(+/-0.6 \%)$ & $56.5 \%(+/-1.2 \%)$ \\
5 & $63.3 \%(+/-1.0 \%)$ & $64.6 \%(+/-1.0 \%)$ & $64.6 \%(+/-1.0 \%)$ & $64.2 \%(+/-1.1 \%)$ \\
6 & $68.0 \%(+/-0.9 \%)$ & $71.5 \%(+/-0.4 \%)$ & $71.5 \%(+/-0.4 \%)$ & $70.3 \%(+/-1.9 \%)$ \\
7 & $62.5 \%(+/-0.9 \%)$ & $62.3 \%(+/-0.8 \%)$ & $62.3 \%(+/-0.8 \%)$ & $62.3 \%(+/-0.7 \%)$ \\
\hline Average $(n=21)$ & $61.9 \%(+/-3.7 \%)$ & $62.9 \%(+/-4.5 \%)$ & $63.5 \%(+/-4.2 \%)$ & $\mathbf{6 2 . 8 \% ( + / - 4 . 2 \% )}$
\end{tabular}

Table 1 expresses the calculated $\mathrm{StO}_{2}$ of the 3 recorded sessions. For each session, the average $\mathrm{StO}_{2}$ value for the 3 repetitions is computed, and the standard deviation (SD) is expressed. The last column of the table shows the average $\mathrm{StO}_{2}$ value over the 3 sessions, leading to $9 \mathrm{StO}_{2}$ values. The last row expresses the $\mathrm{StO}_{2}$ group average. The grand average $\mathrm{StO}_{2}$ value over all days and volunteers $(\mathrm{n}=63)$ is $62.8+/-4.2 \%$. This is in accordance with previously published data ${ }^{7}$. There is no significant variation in the $\mathrm{StO}_{2}$ measurements within a single session nor between the 3 sessions. Indeed, the withinvolunteer $\mathrm{SD}\left(\mathrm{SD}_{\mathrm{w}}\right.$, calculated as in ref 7, using a one-way repeated-measure ANOVA) in session 1, 2 and 3 are $0.89 \%$, $1.10 \%$ and $0.82 \%$ respectively, and the $\mathrm{SD}_{\mathrm{w}}$ for all 3 sessions is $1.43 \%$.

\section{DISCUSSION}

This study with our TD-NIRS instrument demonstrates a good short- and mid-term reproducibility. Moreover, our shortterm reproducibility, is comparable with the one of a previous study by Giacalone and colleagues ${ }^{7}$, who reported a $\mathrm{SD}_{\mathrm{w}}$ of 
$1.8 \%$ for 3 repeated measurements on the left prefrontal cortex of 63 adult volunteers. One limitation in our study was that we performed the measurements in a dark room, which is not representative of the hospital environment. We will continue this work over the following months to see if the measurements are stable over a longer period of time and test the reproducibility in a more realistic environment. The results of this work are important in order to justify the use of our TDNIRS instrument in following up patients over a long period of time. Indeed, most of the brain oximeter that have been tested in the clinic are CW, mainly because of their simplicity. The reproducibility of such system has mostly been investigated on newborn but have produced contrasting results (from 2.4 to $14.8 \%$, data extracted from ref 7). Therefore, even though TD-NIRS appears to be more complicated and bulkier than CW systems, the gain in performance could justify its use.

In conclusion, this study reports absolute values of $\mathrm{StO}_{2}$ comparable to previously published data and provides evidence that TD-NIRS brain measurements are reproducible over a few days. If the reproducibility is good enough, a study looking at long-term variation of cerebral $\mathrm{StO}_{2}$, in response to drugs for example, could be designed.

\section{ACKNOWLEDGEMENTS}

The authors thank The Wellcome Trust (104580/Z/14/Z) for financial support of this work.

\section{REFERENCES}

[1] Bale, G., Elwell, C. E. and Tachtsidis, I., "From Jöbsis to the present day: a review of clinical near-infrared spectroscopy measurements of cerebral cytochrome-c-oxidase," J. Biomed. Opt. 21(9), 091307 (2016).

[2] Obrig, H., "NIRS in clinical neurology - a 'promising' tool?" Neuroimage 85, 535-546 (2014).

[3] Contini, D., Zucchelli, L., Spinelli, L., Caffini, M., Re, R., Pifferi, A., Cubeddu, R. and Torricelli, A., "Review: Brain and muscle near infrared spectroscopy/imaging techniques," J. Near Infrared Spectrosc. 20(1), 15 (2012).

[4] Kovacsova, Z., Bale, G. and Tachtsidis, I., "Medical Utility of NIR Monitoring," [Encyclopedia of Biomedical Engineering], Elsevier, 415-431 (2019).

[5] Yang, R. and Dunn, J. F., "Reduced cortical microvascular oxygenation in multiple sclerosis: a blinded, casecontrolled study using a novel quantitative near-infrared spectroscopy method," Sci. Rep. 5(1), 16477 (2015).

[6] Wabnitz, H., Jelzow, A., Mazurenka, M., Steinkellner, O., Macdonald, R., Milej, D., Zolek, N., Kacprzak, M., Sawosz, P., Maniewski, R., Liebert, A., Magazov, S., Hebden, J., Martelli, F., Di Ninni, P., Zaccanti, G., Torricelli, A., Contini, D., Re, R., et al., "Performance assessment of time-domain optical brain imagers, part 2: nEUROPt protocol.," J. Biomed. Opt. 19(8), 86012 (2014).

[7] Giacalone, G., Zanoletti, M., Contini, D., Re, R., Spinelli, L., Roveri, L. and Torricelli, A., "Cerebral time domainNIRS: reproducibility analysis, optical properties, hemoglobin species and tissue oxygen saturation in a cohort of adult subjects," Biomed. Opt. Express 8(11), 4987 (2017).

[8] Hallacoglu, B., Sassaroli, A., Wysocki, M., Guerrero-Berroa, E., Schnaider Beeri, M., Haroutunian, V., Shaul, M., Rosenberg, I. H., Troen, A. M. and Fantini, S., "Absolute measurement of cerebral optical coefficients, hemoglobin concentration and oxygen saturation in old and young adults with near-infrared spectroscopy.," J. Biomed. Opt. 17(8), 081406-1 (2012).

[9] Lange, F., Dunne, L., Hale, L. and Tachtsidis, I., "MAESTROS: A Multiwavelength Time-Domain NIRS System to Monitor Changes in Oxygenation and Oxidation State of Cytochrome-C-Oxidase," IEEE J. Sel. Top. Quantum Electron. 25(1), 1-12 (2019). 